\title{
ARTIGOS
}

Recebido em 10.04.2012. Aprovado em 12.12.2012

Avaliado pelo sistema double blind review. Editora Científica: Fernanda Finotti Perobelli

\section{NEGOCIAÇÃO COM INFORMAÇÃO PRIVILEGIADA E RETORNO DAS AÇÕES NA BM\&FBOVESPA}

\author{
Informed trading and stock returns in the BM\&FBOVESPA \\ Negociación con información privilegiada y retorno de las acciones en \\ BM\&FBOVESPA
}

\section{RESUMO}

No Brasil, conforme a Lei no $10.303 / 2001$, o uso indevido de informação privilegiada no mercado acionário é crime. Contudo, na literatura, é possível encontrar estudos que apontam a existência de negociações com informação privilegiada nesse mercado (BOPP, 2003; BARBEDO, SILVA, LEAL, 2009). Por isso, este estudo tem o objetivo de investigar a probabilidade de negociação com informação privilegiada (PIN) na negociação de ações na BM\&FBOVESPA, buscando identificar sua relação com o retorno dessas ações. Com base nas Teorias dos Mercados Eficientes e da Agência, foram analisadas 198 ações durante 0 ano de 2011. Por meio do modelo de mensuração de assimetria de informação de Easley, Hvidkjaer e O'Hara (2002), a PIN foi estimada e relacionada ao retorno das ações por meio do modelo de Fama e MacBeth (1973) ajustado. Os resultados indicam que há 22,9\% de probabilidade de terem ocorrido negociações com informação privilegiada, que os segmentos com maiores exigências de governança reúnem as ações com menor assimetria e que um aumento de $10,0 \%$ na PIN leva a um aumento de $8,0 \%$ no retorno das ações.

PALAVRAS-CHAVE | Assimetria de informação, informação privilegiada, insider trading, probability of informed trading, PIN.

\begin{abstract}
In Brazil, according to Law No. 10.303/2001, the improper use of inside information in the stock market is crime. However, in literature there are studies to indicate the existence of insider trading in this market (BOPP, 2003; BARBEDO; SILVA; LEAL, 2009). Therefore, this study aims to investigate the Probability of Informed Trading (PIN) in stock trading on the BM\&FBOVESPA, trying to identify its relationship with stock returns. Based on Efficient Markets Theory and Agency Theory, we analyzed 198 stocks during the year 2011. The information asymmetry was measured (PIN) using the model of Easley, Hvidkjaer and O'Hara (2002) and related to stock returns through the model of Fama and MacBeth (1973) set. The results indicate that there is $22.9 \%$ probability of insider trading has occurred, that the segments with higher governance requirements together with the stocks that lower information asymmetry and an increase of $10.0 \%$ in PIN leads to an increase of $8.0 \%$ in stock returns.
\end{abstract}

KEYWORDS / Information asymmetry, inside information, insider trading, probability of informed trading, PIN.

\section{RESUMEN}

En Brasil, de acuerdo con la Ley no 10.303/2001 el uso indebido de información privilegiada en el mercado de acciones es crimen. Sin embargo, en la literatura, es posible encontrar estudios que apuntan la existencia de negociaciones con información privilegiada en ese mercado (BOPP, 2003; BARBEDO, SILVA, LEAL, 2009). Por ello, este estudio tiene como objetivo investigar la probabilidad de negociación con información privilegiada (PIN) en la negociación de acciones en BM\&FBOVESPA, tratando de identificar su relación con el retorno de esas acciones. Con base en las Teorías de los Mercados Eficientes y de la Agencia, se analizaron 198 acciones durante el 2011. Por medio del modelo de medición de asimetría de información de Easley, Hvidkjaer y O'Hara (2002), PIN se estimó y se relacionó al retorno de las acciones por medio del modelo de Fama y MacBeth (1973) ajustado. Los resultados indican que hay un 22,9\% de probabilidad de que hayan ocurrido negociaciones con información privilegiada, que los segmentos con mayores exigencias de gobierno reúnen las acciones con menor asimetría y que un aumento del 10,0\% en PIN resulta en un aumento del 8,0\% en el retorno de las acciones.

PALABRAS CLAVE I Asimetría de información, información privilegiada, insider trading, probability of informed trading, PIN. 


\section{INTRODUÇÃO}

Há tempos, fatores como a globalização da economia dos países, o crescimento dos mercados de capitais e a maior mobilidade do capital têm provocado significativas modificações no cenário das finanças corporativas e na forma de gestão das empresas que compõem os diversos mercados de capitais. No Brasil, esses fatores têm contribuído para a tão desejada solidez do mercado, apesar de ainda ser possível observar alguns de seus problemas crônicos, como a grande concentração do volume de ações negociadas nas mãos de poucos investidores, que, aliada à baixa liquidez de alguns papéis e a um sistema de regulação não tão eficiente quanto o de mercados desenvolvidos, abre espaço para a manipulação de informações ou uso indevido de informação privada, o que se convencionou chamar, na literatura internacional, de insider trading (DUARTE e YOUNG, 2009).

Uma das principais causas da ocorrência de insider trading é a oportunidade de se auferirem lucros instantâneos com base em informações relevantes ainda não divulgadas ao público em geral. Os principais sujeitos dessa prática são os indivíduos diretamente ligados às decisões estratégicas das corporações, e sua ocorrência está vinculada à utilização de suas posições privilegiadas para a obtenção de informações que possam influenciar os preços e retornos futuros das ações das firmas, antes que essas informações cheguem ao conhecimento do público. Por essa razão, a prática é considerada ilegal, conforme 0 artigo 27 da Lei no 6.385/1976, incluído pela Lei no 10.303/2001, pois configura quebra de fidúcia ou de relação de confiança e sigilo entre controladores e proprietários do capital de uma empresa (BRASIL, 1976, 2001).

Nesse contexto, percebe-se que o tema tem se tornado recorrente em discussões acadêmicas e profissionais na literatura internacional (HEIDLE e HUANG, 2002; CRUCES e KAWAMURA, 2005). Com isso, a assimetria de informação passou a ser objeto de estudo de modelos que buscavam sua identificação e mensuração, como o modelo seminal de Easley e outros (1996), posteriormente aperfeiçoado por Easley, Hvidkjaer e O'Hara (2002), doravante denominado modelo EHO, que estima a probabilidade de negociação com informação privilegiada $(P I N)$, ou probability of informed trading, existente nas transações com uma ação por meio de seus volumes intradiários de negociação. Apesar disso, no Brasil, não se tem a mesma percepção de que esse tema seja largamente discutido, principalmente em se tratando do uso de dados de microestrutura do mercado para essas pesquisas, haja vista a escassez de estudos que abordem essa problemática.

Dessa forma, este estudo surge com a motivação de investigar a PIN no mercado acionário brasileiro, por meio do modelo de Easley e outros (1996) e Easley, Hvidkjaer e O'Hara (2002), que, segundo Heidle e Huang (2002), possui inúmeras aplicações na literatura, destacando-se por apresentar uma proxy para a assimetria de informação mais robusta que as demais (como a volatilidade, os lucros anormais e o número de anúncios públicos sobre a empresa), pois é estimada com base nos dados das negociações das ações. Isso é ratificado por Abad e Rubia (2005), que afirmam que a vantagem da PIN em relação às demais proxies consiste no fato de ela proporcionar uma medida direta de assimetria, mais independente da organização do mercado. Cruces e Kawamura (2005) são ainda mais enfáticos, ao observarem que esse é o único método que permite a estimação direta da assimetria de informação no mercado.

O Brasil possui um mercado de capitais com características propícias à ocorrência de negociações baseadas em informação privada. Entre elas, citam-se: (i) a pouca participação de empresas de outros países, (ii) a concentração do capital das empresas nas mão de poucos investidores, (iii) o pequeno volume de ativos negociados e (iv) o perfil dos investidores pautado no curto prazo (VIEIRA e MENDES, 2006; DUARTE e YOUNG, 2009). Nesse sentido, Barbedo, Silva e Leal (2009) demonstram que a PIN pode ser estimada no mercado brasileiro, ratificando as evidências anteriores de existência de assimetria de informação e sugerindo que os níveis diferenciados de governança corporativa, se considerada a liquidez das ações, podem indicar as firmas com maior ou menor assimetria entre seus controladores e proprietários.

Esse conflito de interesses entre controladores e proprietários, por sua vez, é tratado pela Teoria da Agência (JENSEN e MECKLING, 1976), que vem de encontro à Hipótese dos Mercados Eficientes (HME) de Fama (1970, 1991), em sua forma forte, pois prevê que uma informação privada pode proporcionar uma vantagem competitiva ao seu portador na negociação das ações de uma empresa. Além disso, Easley e outros (1996) destacam que a existência de assimetria de informação no mercado de capitais cria um novo tipo de risco sistemático, o risco de informação, e Easley, Hvidkjaer e O'Hara (2002) alertam que esse tipo de risco é precificado pelo mercado, sendo refletido no retorno das ações.

Nesse sentido, com base em estudos assemelhados, desenvolvidos nos Estados Unidos, por Easley e outros (1996), Easley, Kiefer e O’Hara (1997), Easley, Hvidkjaer e O'Hara (2002) e Nyholm (2002, 2003); na Espanha, por Abad e Rubia (2005); e, no Brasil, por Barbedo, Silva e Leal (2009), este estudo busca responder à seguinte questão de pesquisa: "Qual é a probabilidade de negociação com informação privilegiada das ações negociadas na BM\&FBOVESPA e qual é a sua relação com o retorno dessas ações?". Assim, seu objetivo principal é investigar 
a probabilidade de negociação com informação privilegiada no mercado acionário brasileiro e seu reflexo no retorno das ações. Para tanto, foi realizado um estudo exploratório com base no volume intradiário de negociação de 198 ações listadas na BM\&FBOVESPA durante o ano de 2011. Com isso, durante o período analisado, foi possível observar que há indícios de utilização de informação privilegiada no mercado acionário brasileiro e que esse fenômeno tem influenciado o retorno das ações.

\section{REFERENCIAL TEÓRICO}

A discussão acerca da existência de assimetria de informação em um ambiente institucional foi abordada principalmente pela Teoria da Agência (JENSEN e MECKLING, 1976), indo de encontro à forma forte de eficiência da Teoria dos Mercados Eficientes (FAMA, 1970). Nesse sentido, este estudo fundamenta-se nessas teorias para investigar a assimetria de informação no mercado de capitais brasileiro. Para tanto, apresenta-se o arcabouço do modelo EHO, que busca mensurar essa assimetria, estimando a PIN das ações para que ela possa ser relacionada aos seus retornos.

\section{Hipótese dos Mercados Eficientes e assimetria de informação}

A Hipótese dos Mercados Eficientes (HME) de Fama (1970, 1991) sugere que, em um mercado equilibrado, os preços dos ativos sempre fornecem sinais adequados para a alocação de recursos, isto é, as empresas podem tomar decisões de produção e investimento e os investidores podem escolher ativos que representam a posse dessas empresas, sob a hipótese de que os preços desses ativos sempre refletirão todas as informações relevantes disponíveis. Na HME, em sua forma forte de eficiência, o valor de um ativo é reflexo do consenso dos participantes do mercado, com relação ao seu desempenho esperado, e nenhum investidor é capaz de identificar ativos com preços em desequilíbrio, pois todas as informações relevantes sobre eles estão disponíveis a todos os participantes do mercado. Desse modo, nenhum participante poderia tirar proveito de uma informação privada para aumentar seus ganhos em uma negociação, pois ela já estaria refletida nos preços das ações.

Nesse contexto, a informação privada, ou privilegiada, é aquela que ainda não foi divulgada ao público em geral, a qual pode proporcionar vantagens competitivas ao seu portador na negociação das ações de uma firma. Ela é consequência da assimetria de informação existente entre os gestores e os acionistas da empresa, fenômeno tratado pela Teoria da Agência
(JENSEN e MECKLING, 1976), que se apresenta como arcabouço teórico voltado para a análise da relação entre os participantes de uma firma, em que a propriedade e o controle do capital são destinados a indivíduos distintos, dando espaço à formação de conflito de interesses. Assim, essa relação é classificada como uma relação de agência, na qual principal ou agente pode tirar proveito de uma vantagem informacional em benefício próprio.

A Teoria da Agência trata essa diferença informacional existente entre os agentes do mercado sob dois aspectos. 0 primeiro, no que diz respeito à seleção adversa, a partir do momento em que um investidor externo não consegue determinar o valor fundamental dos ativos da firma, devido ao desconhecimento de informações privadas que deveriam ter sido divulgadas ao público. 0 segundo, no que diz respeito ao risco moral, por meio do comportamento oportunista do investidor interno, que se aproveita de sua posição funcional e do acesso privilegiado que tem a determinadas informações e as utiliza em suas negociações, com o objetivo de desfrutar melhores resultados para si mesmo.

Nesse sentido, Albanez e Valle (2009) observam que tanto a seleção adversa quanto o risco moral são considerados falhas de mercado que surgem da assimetria de informação existente entre seus agentes. Para Easley e outros (1996) e Easley, Hvidkjaer e O’Hara (2002), essa assimetria cria um novo tipo de risco sistemático, denominado risco de informação, que apresenta correlação positiva com o retorno das ações. Aliado a isso, Abad e Rubia (2005) atentam que a existência de assimetria de informação entre os investidores de um mercado tem provocado profundas complicações à liquidez dos ativos nele negociados e, em termos gerais, tem se tornado essencial para o entendimento do mecanismo de ajuste de preços desses ativos. Todavia, no Brasil, estudos que busquem mensurar a assimetria de informação no mercado de capitais e seu reflexo nos preços desses ativos são escassos. Por isso, este estudo surge com a motivação de preencher essa lacuna na literatura nacional, com base na mensuração da assimetria de informação existente no mercado acionário brasileiro.

\section{Probabilidade de negociação com informação privilegiada e retorno das ações}

A probabilidade de negociação com informação privilegiada, tratada na literatura internacional como probability of informed trading, ou simplesmente PIN, é o resultado da mensuração da assimetria informacional existente no mercado de capitais. Ela é estimada com base nos dados de microestrutura desse mercado, por meio de um modelo de negociação sequencial que busca modelar explicitamente o processo de ne- 
gociação de uma ação, por meio de uma inferência bayesiana. Essa probabilidade foi modelada inicialmente por Easley e outros (1996), com base nos trabalhos teóricos de Easley e O’Hara $(1987,1992)$. Esse modelo apresenta uma versão aperfeiçoada por Easley, Hvidkjaer e O'Hara (2002), denominada modelo EHO, a qual é utilizada neste estudo, pois considera a assimetria de informação um tipo de risco sistemático.

Esse modelo parte do pressuposto de que eventos informacionais podem surgir durante o dia de negociação, quando uma informação que chega ao mercado pode ser uma "boa notícia”, indicando um sinal de alta do valor do ativo, ou uma "má notícia", indicando um sinal de baixa. Assim, mais ordens de compra desse ativo são esperadas em dias nos quais boas notícias predominem, mais ordens de venda são esperadas em dias nos quais más notícias prevaleçam, enquanto poucas negociações são esperadas em dias sem eventos informacionais, devido à diminuição de negociadores informados no mercado. Com isso, o desequilíbrio observado entre os volumes de ordens de compra ou venda revela a presença de negociadores informados, participando mais ativamente de um dos lados do mercado, indicando a PIN.

No modelo EHO, a probabilidade de que um evento informacional tenha ocorrido antes do início do pregão é a, enquanto a probabilidade de que ele não tenha surgido é $1-\alpha$. Aliado a isso, o ativo negociado tem um valor $(V)$ no final do dia de negociação. A ocorrência de um evento informacional é representada pela chegada de um sinal $\Psi$ sobre $V$. Esse evento, por sua vez, pode assumir dois valores, baixo $(L)$ ou alto $(H)$, com probabilidades $\delta$ e $1-\delta$. O valor do ativo condicionado ao sinal de baixa $(L)$, indicando má notícia, é dado por $\underline{V}$; similarmente, aquele condicionado ao sinal de alta $(H)$, boa notícia, é dado por $\bar{V}$. Eventos informacionais também podem não ocorrer, refletindo o fato de que novas informações não surgiram durante o período de negociação. Se nenhum evento ocorrer, assumese que $\Psi=0$ e que o valor do ativo continua a ser, em seu nível incondicional, $V=\delta \underline{V}+(1-\delta) \bar{V}$, onde $\underline{V}<V<\bar{V}$ (EASLEY e outros, 1996; EASLEY, KIEFER, O’HARA, 1997).

\section{Figura 1. Diagrama de árvore do processo de negociação}

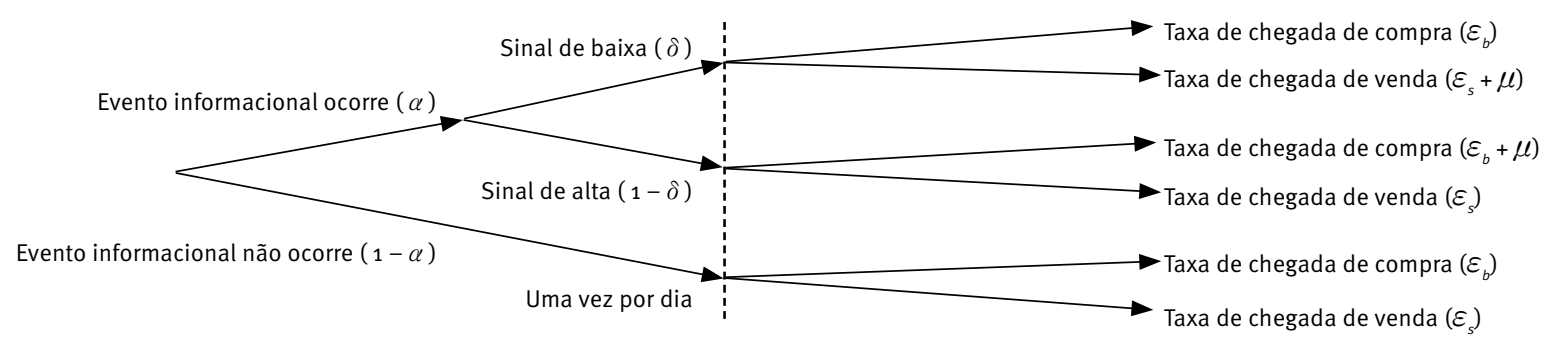

Fonte: Adaptado de Easley, Hvidkjaer e O’Hara (2002, p. 2196). dos. Sua estratégia de negociação é: se observar um sinal de alta, ele vai comprar as ações, se a cotação atual estiver abaixo de $\bar{V}$; se observar um sinal de baixa, ele vai vender, se a cotação estiver acima de $\underline{V}$. Já os negociadores desinformados comercializam apenas por motivos de liquidez, pois não possuem informações que orientem seus negócios (EASLEY, KIEFER, O'HARA, 1997). Assim, é razoável esperar que os negociadores desinformados possuam demandas que dependam do histórico e das cotações das ações, considerando que seus preços estão condicionados aos valores esperados. Por isso, assume-se que a probabilidade de eles negociarem ao verificar a cotação é $\varepsilon>0$.

Nesse processo, as negociações ocorrem sequencialmente, o que significa que, em cada intervalo de tempo, algum negociador é escolhido aleatoriamente, de acordo com as probabilidades e oportunidades dadas ao negócio. Em cada tempo $t[0, T]$, o formador de mercado define os preços para compra ou venda durante o dia e, em seguida, executa as ordens que chegam. Negociadores informados com más notícias $(\delta)$ sobre um ativo o vendem, enquanto aqueles informados com boas notícias $(1-\delta)$ o compram. Com isso, em dias com eventos informacionais, ordens de negociadores informadores chegam a uma taxa $\mu$. Já os negociadores desinformados comercializam por motivos de liquidez. Assim, ordens de compradores desinformados chegam a uma taxa $\varepsilon_{b}$ e ordens de vendedores desinformados chegam a uma taxa $\varepsilon_{s}$. Se uma ordem chega no tempo $t$, o formador do mercado observa a negociação (seja compra ou venda) e usa essa informação para atualizar suas crenças. Assim, novos preços são fixados, as negociações evoluem e os preços movem-se em resposta às mudanças das crenças do formador de mercado (EASLEY, HVIDKJAER, O'HARA, 2002).

Esse processo de negociação é demonstrado em um diagrama de árvore, conforme a Figura 1. 
No diagrama de árvore, os nós à esquerda da linha tracejada ocorrem somente uma vez por dia, ao passo que os nós à direita dessa linha podem ocorrer a cada intervalo de tempo. Assim, o diagrama demonstra a relação existente entre a informação, quando boa ou má, e sua probabilidade de influência na negociação. 0 primeiro nó corresponde à ocorrência ou não de um evento informacional. Se o evento ocorre (com probabilidade $\alpha$ ), então o tipo de sinal é determinado no segundo nó. Há uma probabilidade $\delta$ de o evento ocasionar um sinal de baixa e uma probabilidade $1-\delta$ de ocasionar um sinal de alta. Dessa forma, o terceiro nó é atingido no início do dia de negociação, refletindo o pressuposto do modelo de que os eventos informacionais ocorrem apenas uma vez ao dia.

A partir desse ponto, os negociadores são selecionados em cada tempo t para operações com base nas probabilidades descritas anteriormente. Se um evento informacional ocorre, então se segue para a parte superior da árvore, e um agente informado é escolhido para negociar com probabilidade $\mu$. Se ele compra ou vende, depende do sinal que ele vê. Se o evento informacional ocorre indicando um sinal de baixa, a taxa de chegada de ordens de compra é dada por $\varepsilon_{b}+\mu$. Se o evento ocorre e indica um sinal de alta, a taxa de chegada de ordens de venda é dada por $\varepsilon_{s}+\mu$. Mas, se o evento informacional não ocorre, as taxas de chegada de ordens de compras e de vendas desinformadas são dadas por $\varepsilon_{b}$ e $\varepsilon_{s}$, respectivamente.

Isso permite ao modelo EHO a utilização de dados observáveis sobre o volume de ordens de compras e de vendas diárias para fazer inferências sobre eventos informacionais não observáveis, além de distinção entre negociação informada (insider trading) e desinformada. Dessa forma, o modelo interpreta o volume padrão de compras e vendas de uma ação como uma negociação desinformada, utilizando esses dados para identificar $\varepsilon_{b}$ e $\varepsilon_{s}$. Um volume anormal de ordens de compras ou vendas é interpretado como negociação informada e é utilizado para identificar $\mu$. Por outro lado, o número de dias em que há volume anormal de compras e vendas é utilizado para identificar $\alpha$ e $\delta$ (EASLEY, HVIDKJAER, O'HARA, 2002).

Sendo assim, os parâmetros do modelo $\operatorname{EHO}\left(\alpha, \mu, \varepsilon_{b}, \varepsilon_{s}, \delta\right)$ podem ser estimados por meio da maximização de uma função de máxima verossimilhança condicionada ao histórico de negociação das ações, conforme Equação 1.

$$
\begin{aligned}
L(\theta \mid B, S) & =(1-\alpha) e^{-\varepsilon_{b}} \frac{\varepsilon_{b}^{B}}{B !} e^{-\varepsilon_{s}} \frac{\varepsilon_{s}^{S}}{S !} \\
& +\alpha \delta e^{-\varepsilon_{b}} \frac{\varepsilon_{b}^{B}}{B !} e^{-\left(\mu+\varepsilon_{s}\right)} \frac{\left(\mu+\varepsilon_{s}\right)^{s}}{S !} \\
& +\alpha(1-\delta) e^{-\left(\mu+\varepsilon_{b}\right)} \frac{\left(\mu+\varepsilon_{b}\right)^{B}}{B !} e^{-\varepsilon_{s}} \frac{\varepsilon_{s}^{S}}{S !}
\end{aligned}
$$

Em que $B$ e $S$ representam o volume de compras e vendas da ação $i$ em um dia de negociação $t$, respectivamente, e $\theta=(\alpha$, $\left.\mu, \varepsilon_{b}, \varepsilon_{s}, \delta\right)$ é o vetor de parâmetros. Essa probabilidade é uma mistura de distribuições em que os resultados das negociações são ponderados pela probabilidade de ser uma boa notícia $\alpha(1-\delta)$, uma má notícia $(\alpha \delta)$ ou de não haver notícia (1 - $\alpha)$. Evidentemente, o risco da ocorrência de negociação baseada em informação envolve todos esses elementos de maneira simultânea, como demonstra a Equação 2.

$$
P I N=\frac{\alpha \mu}{\alpha \mu+\varepsilon_{b}+\varepsilon_{s}}
$$

Em que $\alpha$ é a probabilidade de ocorrer um evento informacional; $\mu$ é a taxa de chegada de ordens de negociadores informados; $\varepsilon_{b}$ é a taxa de chegada de ordens de compradores desinformados; e $\varepsilon_{s}$ é a taxa de chegada de ordens de vendedores desinformados. Dessa forma, $\alpha \mu+\varepsilon_{b}+\varepsilon_{s}$ é a taxa de chegada de todas as ordens e $\alpha \mu$ é a taxa de chegada de ordens baseadas em informações. A razão é, portanto, a fração de ordens que surge de negociadores informados ou a probabilidade de negociação com informação privilegiada $(P I N)$.

Nesse sentido, Easley, Hvidkjaer e O’Hara (2002, 2010) afirmam que há indícios de pagamento de um prêmio no mercado pela existência de risco de informação, uma vez que o aumento da PIN tende a proporcionar aumento do excesso de retorno das 
ações. Embora Fama (1970) tenha sugerido, em sua forma forte de eficiência, que não deva ser pago prêmio pelo risco de informação, pois ele seria potencialmente diversificável, Easley, Hvidkjaer e O’Hara (2002) afirmam que, no mercado norte -americano, um aumento de cerca de $10,0 \%$ na PIN proporciona um aumento médio de $2,5 \%$ no retorno esperado das ações. No mercado brasileiro, não foi encontrado estudo com essa abordagem, mas Camargos e Barbosa (2003) observam que as pesquisas empíricas têm encontrado suporte apenas para as formas de eficiência fraca e semiforte, enquanto a maioria dos estudos rejeita a forma forte de eficiência. Nesse contexto, Barbedo, Silva e Leal (2009) demonstram que a PIN pode ser estimada no mercado acionário brasileiro, contudo não a relacionam ao retorno das ações. Assim, este estudo busca contribuir com a literatura nacional, preenchendo essa lacuna existente.
A população objeto de análise deste estudo foi composta pelas ações das empresas abertas listadas na principal bolsa de valores do Brasil, a BM\&FBOVESPA. Para evitar o problema de viés de seleção na definição da amostra de ações analisada, foram coletados os volumes intradiários de negociação e as informações financeiras de todas as ações negociadas durante o ano de 2011, com base na plataforma de negociação eletrônica CMA Series 4, do grupo CMA, e no banco de dados da Economatica, respectivamente. Apenas após a coleta de todos os dados necessários, foi definida a amostra, composta por ações ativas e que tenham apresentado pelo menos um negócio em cada um dos dias de negociação do período analisado. Esse procedimento foi adotado para garantir a robustez das estimativas da PIN pelo modelo EHO. Com isso, a amostra contou com 198 ações de 178 empresas, como demonstra a Tabela 1.

\section{PROCEDIMENTOS METODOLÓGICOS}

\section{TABELA 1. Ações componentes da amostra, conforme segmento de governança - 2011}

\begin{tabular}{l|c|c}
\hline Segmento & Ações listadas* & Ações analisadas \\
\hline Tradicional & 303 & 45 \\
\hline Nivel 1 & 63 & 36 \\
\hline Nível 2 & 34 & 98 \\
\hline Novo Mercado & 123 & 98 \\
\hline Total & 523 & 198
\end{tabular}

Nota: * Não foram consideradas na população as ações das companhias listadas no Bovespa Mais e no Balcão Organizado Tradicional.

\section{Coleta e tratamento dos dados}

Para a estimação dos parâmetros do modelo EHO, foram coletados os volumes intradiários das 198 ações analisadas durante os 249 dias nos quais houve negociações no ano de 2011. Com isso, foi possível estimar a PIN para cada trimestre de 2011 , tendo em vista que eles apresentaram mais de 60 dias de negociação, intervalo necessário para uma estimação consistente da PIN (EASLEY e outros, 1996). Sendo assim, essas informações foram obtidas em alta frequência, minuto a minuto, com base no banco de dados da plataforma de negociação eletrônica CMA Series 4. Já as demais informações patrimoniais e financeiras das firmas foram obtidas no banco de dados da Economatica.
Como o CMA não identifica se os negócios intradiários partiram de um agente comprador ou vendedor, as operações foram classificadas em compra ou venda com base no algoritmo de Lee e Ready (1991), largamente utilizado na literatura internacional (EASLEY e outros, 1996; EASLEY, KIEFER, O'HARA, 1997; EASLEY, HVIDKJAER, O'HARA, 2002; EASLEY, HVIDKJAER, O'HARA, 2010; ABAD e RUBIA, 2005; CRUCES e KAWAMURA, 2005; DUARTE e YOUNG, 2009). Assim, para todo negócio, o preço da transação foi comparado com as melhores ofertas de compra e venda do formador de mercado. Se o preço da transação estava abaixo do ponto médio entre os preços de compra e venda, a transação foi classificada como "venda"; analogamente, se estava acima do ponto médio, foi classificada como "compra". 


\section{Estimativa dos parâmetros do modelo EHO}

A maximização da Equação 1 é uma tarefa difícil até mesmo para um computador, pois, se houver um grande número de compras $(B)$ e vendas $(S)$ diárias para cada ação, o sistema estoura, devido à fatoração das variáveis $B$ e $S$, que pode gerar números infinitos, o que acontece com nossa amostra. Sendo assim, de acordo com Easley, Hvidkjaer e O'Hara (2010), essa equação pode ser reescrita conforme a Equação 3.

$$
\begin{aligned}
& \left.L\left(\left(B_{t}, S_{t}\right)_{t=1}^{T}\right) / \theta\right)= \\
& \sum_{t=1}^{T}\left[-\varepsilon_{b}-\varepsilon_{s}+M_{t}\left(\ln x_{b}+\ln x_{s}\right)+B_{t} \ln \left(\mu+\varepsilon_{b}\right)+S_{t} \ln \left(\mu+\varepsilon_{s}\right)\right]+ \\
& \sum_{t=1}^{T} \ln \left[\alpha(1-\delta) e^{-\mu} X_{s}^{S_{t}-M_{t}} X_{b}^{-M_{t}}+\alpha \delta e^{-\mu} X_{b}^{B_{t}-M_{t}} X_{s}^{-M_{t}}+(1-\alpha) X_{s}^{S_{t}-M_{t}} X_{b}^{B_{t}-M_{t}}\right]
\end{aligned}
$$

Em que $M_{t}=\min \left(B_{t}, S_{t}\right)+\max \left(B_{t}, S_{t}\right) / 2, x_{s}=\varepsilon_{s} /\left(\mu+\varepsilon_{s}\right)$ e $x_{b}=\varepsilon_{b} /\left(\mu+\varepsilon_{b}\right)$. De acordo com Easley, Hvidkjaer e O'Hara (2010), a fatoração de $X_{b}^{M_{t}}$ e $X_{s}^{M_{t}}$ é feita para reduzir o erro de truncamento e aumentar a eficiência do sistema. Para os autores, isso é importante para populações com grandes volumes de compras e vendas. Essa transformação permite o cálculo da PIN para um número maior de dias de negociação, porque elimina os grandes números, como os fatoriais dos números de ordens de compras (B!) e vendas (S!), sem, no entanto, prejudicar o processo de estimação. Dessa forma, os parâmetros do modelo EHO e a própria PIN foram estimados para cada ação, de modo trimestral. Além disso, devido ao grande número de iterações necessárias, foi utilizada uma rotina desenvolvida no software estatístico "R", que é um software livre, de código aberto.

\section{Relação da PIN com o retorno}

Para investigar a relação da PIN com o retorno das ações, foi estimada a Equação 4, com dados trimestrais em cross-section, baseada no modelo de apreçamento de ativos de Fama e Macbeth (1973), adaptado por Easley, Hvidkjaer e O’Hara (2002) com a inclusão da variável PIN.

$$
R_{i t}=\gamma_{0 t}+\gamma_{1 t} \beta_{i t}+\gamma_{2 t} P N_{i t-1}+\gamma_{3 t} \operatorname{Tam}_{i t-1}+\gamma_{4 t} B M_{i t-1}+\eta_{i t}
$$

Em que $R_{i t}$ é o excesso de retorno da ação $i$ no trimestre $t$, representado pela diferença entre o retorno da ação e o retorno do mercado, representado pelo Índice Bovespa (Ibovespa); $\gamma_{j t}, j=1, \ldots 4$, são os coeficientes estimados; $\beta_{i t}$ é o beta da ação $i$ no trimestre $t$, que é dado pela covariância do retorno da ação com o retorno do mercado, dividida pela variância do retorno do mercado; $P I N_{i t-1}$ é a PIN da ação $i$ no trimestre $t$-1; Tam ${ }_{i t-1}$ é o logaritmo do valor de mercado da empresa $i$ no final do trimestre $t$-1; $B M_{i t-1}$ é o logaritmo do índice book-to-market da empresa $i$ no trimestre $t$-1; e, $\eta_{i t}$ é o termo de erro.

Além disso, para aproximar o referido modelo das características do mercado de capitais brasileiro, foi considerada a observação de Duarte e Young (2009) de que algumas características dos mercados de capitais de países emergentes estão associadas à maior assimetria de informação, como a baixa liquidez e a alta volatilidade de alguns papéis. Aliado a isso, destaca-se a afirmação de Easley, Hvidkjaer e O’Hara (2002) de que maior assimetria está relacionada a maior retorno. Dessa forma, foram adicionadas ao modelo proposto por esses autores duas variáveis: liquidez e volatilidade.

$$
R_{i t}=\gamma_{0 t}+\gamma_{1 t} \beta_{i t}+\gamma_{2 t} P N_{i t-1}+\gamma_{3 t} \operatorname{Tam}_{i t-1}+\gamma_{4 t} B M_{i t-1}+\gamma_{s t} L_{i q_{i t-1}}+\gamma_{6 t} \text { Volat }_{i t-1}+\eta_{i t}
$$


A primeira variável adicionada foi a liquidez em bolsa. Segundo Duarte e Young (2009), a liquidez de uma ação está diretamente relacionada às suas informações disponíveis, pois quanto mais informações existirem, menor é o risco de informação da ação. Por isso, medidas de liquidez e de assimetria de informação podem ser correlacionadas, pois quanto maior a assimetria de informação, maior é o risco inerente a essa ação e, consequentemente, maior seu retorno esperado. Sendo assim, a liquidez foi estimada conforme a Equação 6.

$$
L i q_{i t-1}=100 \times \frac{p}{p} \times \sqrt{\frac{n}{N} \times \frac{V}{V}}
$$

Em que $L i q_{i t-1}$ é a liquidez da ação $i$ no trimestre $t$-1; $p$ é o número de dias em que houve, pelo menos, um negócio com a ação $i$ no trimestre $t$-1; $P$ é o número total de dias do trimestre $t$-1; $n$ é o número de negócios com a ação $i$ no trimestre $t-1 ; N$ é o número de negócios com todas as ações no trimestre $t$-1; $v$ é o volume financeiro da ação $i$ no trimestre $t$-1; e $V$ é o volume financeiro de todas as ações no trimestre $t$-1.

A segunda variável adicionada ao modelo foi a volatilidade, que, para Halov e Heider (2011), pode ser vista como uma medida de assimetria informacional, pois a alta volatilidade de uma ação pode indicar risco ao investidor externo, que, por sua vez, exige maior retorno para seu investimento. Neste estudo, a volatilidade foi estimada por meio da Equação 7.

$$
\text { Volat }_{i t-1}=\sqrt{\frac{\sum\left(S_{i}-S_{m}\right)^{2}}{n \times P P A}}
$$

Em que Volat ${ }_{i t-1}$ é a volatilidade da ação $i$ no trimestre $t-1$; $S_{i}$ é o logaritmo da cotação da ação $i$ no trimestre $t-1\left(d_{i} / d_{i-1}\right), i=$ $1, \ldots, n ; S_{m}$ é a média de $S_{1}, S_{2}, S_{3}, \ldots, S_{n}$; e $P P A$ é a quantidade de períodos de fechamento dos preços da ação.

\section{RESULTADOS}

Os resultados deste estudo estão divididos em duas partes: na primeira, apresenta-se a análise descritiva da PIN e, na segunda, as relações do retorno das ações com o beta, a PIN, o tamanho da firma, o índice book-to-market, a liquidez e a volatilidade.

\section{PIN estimada para as empresas integrantes da amostra}

A probabilidade de negociação com informação privilegiada (PIN) estimada para o conjunto de ações analisadas é apresentada na Tabela 2. De acordo com os resultados, pode-se verificar que, durante o ano de 2011, houve uma probabilidade média de ocorrência de eventos informacionais $(\alpha)$ de cerca de $53,0 \%$. No que diz respeito ao conteúdo informacional desses eventos, $50,8 \%$ indicam sinais de baixa, isto é, a probabilidade de serem más notícias (contra 49,2\% de serem boas notícias). Com base nisso, pode-se apurar uma PIN média de 22,9\% para as ações analisadas, o que sugere que há indícios de utilização de informação privilegiada na amostra analisada, ratificando as suspeitas de existência de assimetria de informação no mercado de capitais brasileiro. Essa probabilidade, por sua vez, se aproxima do valor apurado por Bopp (2003) para os American Depositary Receipt (ADRs) de empresas brasileiras na NYSE, que foi de $23,9 \%$. Por outro lado, é superior às evidências de Barbedo, Silva e Leal (2009) para 48 ações negociadas na BM\&FBOVESPA, cuja PIN média foi de $12,5 \%$.

TABELA 2. Estatísticas descritivas dos parâmetros do modelo EHO e da PIN - 2011

\begin{tabular}{l|c|c|c|c|c|c}
\hline \multicolumn{1}{c|}{ Estatística } & $\alpha$ & $\mu$ & $\varepsilon_{b}$ & $\varepsilon_{s}$ & $\delta$ & $P I N$ \\
\hline Média & 0,530 & 69,800 & 72,677 & 71,134 & 0,508 & 0,229 \\
\hline Mediana & 0,424 & 66,704 & 53,048 & 53,946 & 0,502 & 0,224 \\
\hline Máximo & 1,000 & 193,134 & 225,782 & 236,361 & 1,000 & 0,674 \\
\hline Mínimo & 0,000 & 3,974 & 0,000 & 0,000 & 0,000 & 0,000 \\
\hline Desvio padrão & 0,286 & 31,394 & 54,347 & 51,907 & 0,321 & 0,067 \\
\hline
\end{tabular}

Durante esse período, a menor PIN estimada para uma ação, em um trimestre, foi nula, sugerindo a inexistência de negociação com informação privada para essa ação, enquanto a maior PIN estimada para uma ação foi $67,4 \%$, indicando que a maior parte das negociações com essa ação pode ter sido orientada por informações privadas. 
Em se tratando das taxas de chegada de ordens de negociação, nota-se que a taxa média de chegada de ordens de negociação de agentes informados ( $\mu$ ) foi de 66,7, demonstrando que, nos dias em que houve negociação com informação privada, o mercado era composto, em sua maioria, por agentes informados. Por outro lado, a taxa média de chegada de ordens de compradores desinformados $\left(\varepsilon_{b}\right)$ foi de 53,0 e a taxa média de chegada de ordens de vendedores desinformados $\left(\varepsilon_{s}\right)$ foi de cerca de 53,9, indicando que, nos dias em que não ocorreram negociações baseadas em informação, o mercado apresentava equilíbrio entre compradores e vendedores sem informação privada, o que se considera a situação ideal para o bom funcionamento do mercado de capitais (EASLEY e outros, 1996; EASLEY, HVIDKJAER, O’HARA, 2002).

No que diz respeito à listagem nos diferentes segmentos de governança corporativa, nota-se que as ações que apresentaram menor PIN média foram aquelas listadas no Novo Mercado, segmento caracterizado pela maior exigência de transparência por parte das firmas, com média de $22,3 \%$, conforme a Tabela 3. Segundo Vieira e Mendes (2006), falar em governança corporativa significa discutir a minimização da assimetria de informação existente entre a empresa e seus diversos agentes, como acionistas, credores, fornecedores e empregados. Por isso, espera-se que a assimetria diminua ao passo que a empresa aumente seu nível de governança corporativa.

Com base nisso, percebe-se que as evidências deste estudo ratificam essa observação, no que diz respeito ao Novo Mercado (22,3\%) e ao Nível 2 (22,5\%), que possuem maiores exigências de práticas diferenciadas de governança. Por outro lado, o mesmo não se pode afirmar para o mercado tradicional (23,4\%) e para o Nível 1 (24,2\%), pois esse último apresentou maior PIN média, o que sugere maior assimetria de informação. Parte-se do pressuposto de que as ações listadas no Nível 1 deveriam apresentar menor PIN, por adotarem práticas diferenciadas de governança corporativa, o que, a priori, não é feito pelas ações listadas no mercado tradicional. No estudo de Barbedo, Silva e Leal (2009), os autores concluíram que a liquidez no mercado brasileiro é influente na determinação da PIN. Assim, quando considerada a liquidez, entre as 48 ações analisadas pelos autores, o Novo Mercado apresentou menor PIN que o Nível 2, assim como o Nível 1 teve PIN média menor que o tradicional.

\section{TABELA 3. Médias dos parâmetros estimados e da PIN, por segmento de governança - 2011}

\begin{tabular}{l|c|c|c|c|c|c}
\hline \multicolumn{1}{c|}{ Segmento } & $\alpha$ & $\mu$ & $\varepsilon_{\boldsymbol{b}}$ & $\varepsilon_{\boldsymbol{s}}$ & $\delta$ & $P I N$ \\
\hline Tradicional & 0,383 & 54,334 & 38,725 & 46,156 & 0,387 & 0,234 \\
\hline Nível 1 & 0,675 & 82,224 & 99,105 & 89,309 & 0,558 & 0,242 \\
\hline Nível 2 & 0,466 & 61,745 & 60,321 & 53,525 & 0,524 & 0,225 \\
\hline Novo Mercado & 0,558 & 73,900 & 80,955 & 79,341 & 0,542 & 0,223 \\
\hline
\end{tabular}

Para verificar se as diferenças entre as médias da PIN de cada segmento são significantes, foi realizado o teste não paramétrico de comparação de médias de Mann-Witney, uma vez que a variável $P I N$ é restrita ao intervalo [0, 1], ou [0\%, 100\%], o que viola a condição de normalidade da maioria dos testes tradicionais. Isso pode ser confirmado pelo teste de Kolmogorov-Smirnov, rejeitando-se, a 1\%, a hipótese nula de distribuição normal dessa variável. Sendo assim, segregando as ações em grupos distintos por nível de governança corporativa, pode-se verificar que a diferença de médias da PIN entre as ações do Novo Mercado e dos demais segmentos (tradicional, Nível 1 e Nível 2) é estatisticamente significante, o que indica que esse segmento realmente reuniu as ações com menor assimetria de informação. Por outro lado, a diferença entre as médias da PIN do mercado tradicional e dos níveis diferenciados (Nível 1, Nível 2 e Novo Mercado) não é significante, como demonstra a Tabela 4.

TABELA 4. Teste de Mann-Whitney de comparação de médias, por segmento de governança - 2011

\begin{tabular}{|c|c|c|}
\hline Segmentos & Z & Significância \\
\hline
\end{tabular}

Nota: ${ }^{\star}$ Significante a $5 \%$.

Tendo como referência a PIN média de 19,1\% encontrada por Easley, Hvidkjaer e O’Hara (2002) para o mercado norte-americano, Bopp (2003) estimou a PIN média para ADRs de empresas latino-americanas negociadas na NYSE, agrupando-as por país. 
Com base nos resultados de Bopp (2003), nota-se que os ADRs de empresas brasileiras apresentaram a menor PIN média da América Latina (23,9\%). Ao compararmos essa média com a PIN estimada neste estudo (22,9\%) para as ações analisadas, nota-se que, pelo menos para essa amostra, a prática de negociação com informação privada no mercado de capitais brasileiro é semelhante àquela apurada para os títulos de empresas brasileiras negociados no mercado norte-americano há cerca de uma década. Ainda, nota-se que essa PIN é inferior àquela apurada para ADRs de outros países da América Latina em 2001, embora continue superior à estimada para os títulos de empresas norte-americanas por Easley, Hvidkjaer e O’Hara (2002), como pode ser observado na Tabela 5 .

\section{TABELA 5. Comparação da PIN média estimada, por país}

\begin{tabular}{|c|c|c|c|c|c|}
\hline País & Média & Mediana & Máximo & Mínimo & Desvio padrão \\
\hline Estados Unidos* & 0,191 & 0,185 & - & - & 0,057 \\
\hline Argentina** & 0,268 & 0,236 & 0,370 & 0,170 & 0,082 \\
\hline Brasil (2001)** & 0,239 & 0,205 & 0,690 & 0,080 & 0,124 \\
\hline Brasil (2011) & 0,229 & 0,224 & 0,674 & 0,000 & 0,067 \\
\hline Chile $^{\star \star}$ & 0,315 & 0,302 & 0,510 & 0,140 & 0,096 \\
\hline Colômbia** & 0,353 & 0,353 & 0,000 & 0,350 & 0,350 \\
\hline México** & 0,267 & 0,257 & 0,510 & 0,130 & 0,083 \\
\hline Peru $^{\star \star}$ & 0,265 & 0,277 & 0,290 & 0,230 & 0,033 \\
\hline Venezuela** & 0,280 & 0,280 & 0,400 & 0,160 & 0,166 \\
\hline
\end{tabular}

Fonte: Adaptado de *Easley, Hvidkjaer e O’Hara (2002) e de **Bopp (2003).

\section{Relação da PIN com o retorno das ações}

A Tabela 6 apresenta a estrutura de correlações entre as variáveis: retorno, beta, PIN, tamanho, book-to-market, liquidez e volatilidade. De acordo com os resultados, as variáveis beta, tamanho, book-to-market e volatilidade apresentaram correlações estatisticamente significantes com o retorno. 0 beta apresentou relação negativa $(-0,174)$ com o retorno, assim como a volatilidade $(-0,184)$, diferentemente do esperado, pois, normalmente, para maior risco se espera maior retorno. Por outro lado, o tamanho apresentou relação positiva com o retorno $(0,107)$ e o book-to-market $(-0,208)$ uma relação negativa, assim como esperado.

\section{TABELA 6. Correlações entre as variáveis analisadas - 2011}

\begin{tabular}{|c|c|c|c|c|c|c|}
\hline & $\beta$ & PIN & TAM & $B M$ & $L I Q$ & VOLAT \\
\hline PIN & & & $0,197^{\star}$ & 0,042 & $0,183^{*}$ & $-0,003$ \\
\hline TAM & & & & $-0,207^{\star}$ & $0,705^{*}$ & $-0,341^{*}$ \\
\hline LIQ & & & & & & $-0,118^{\star}$ \\
\hline
\end{tabular}

Nota: * Significante a $1 \%$.

Em se tratando da $P I N$, sua correlação com o retorno foi positiva (o,065), embora não significante. Com base nas evidências de Easley, Hvidkjaer e O’Hara (2002) no mercado norte-americano, essa relação era esperada, tendo em vista que há uma tendência de que o retorno aumente com o crescimento da PIN. Ainda, a correlação da liquidez com o retorno, apesar de baixa, também foi positiva (0,012), como esperado. 
Nesse contexto, para medir de maneira mais precisa o efeito de cada variável sobre o retorno, foram estimadas duas regressões com correção de White para heterocedasticidade, para garantir a robustez dos parâmetros estimados. Ainda, foi realizado o teste de Durbin-Watson para testar a hipótese nula de ausência de autocorrelação, a qual não foi rejeitada em ambos os modelos. Assim, com base no modelo Fama e MacBeth (1973), a primeira regressão repetiu o modelo de Easley, Hvidkjaer e O’Hara (2002) e a segunda incluiu as variáveis liquidez e volatilidade, para aproximar esse modelo às características do mercado brasileiro. Com isso, espera-se que a PIN apresente coeficiente médio positivo e significante.

A Tabela 7 apresenta os parâmetros estimados nos dois modelos, significantes de acordo com suas estatísticas $F$. 0 primeiro modelo, de Easley, Hvidkjaer e O'Hara (2002) e denominado “original”, apresentou um coeficiente de determinação
$\left(R^{2}\right)$ de 0,054, indicando que aproximadamente 5,4\% das variações observadas no retorno podem ser explicadas pelas variáveis que o compõem. Nesse modelo, o beta e o book-to-market foram significantes a $1 \%$ e a PIN foi significante a $5 \%$, enquanto o tamanho não foi significante na determinação da variável explicada retorno. Destaque seja dado à variável $P I N$, cujo coeficiente $(0,168)$ positivo e significante ratificam a suspeita de que o mercado de capitais brasileiro oferece um prêmio pelo risco de informação, uma vez que um aumento de $10,0 \%$ na PIN ocasionou um aumento no retorno de aproximadamente $1,68 \%$ no trimestre, ou 6,9\% no ano. Esse valor é superior ao prêmio encontrado por Easley, Hvidkjaer e O’Hara (2002) no mercado norte-americano ( $2,5 \%$ no ano), embora inferior ao encontrado por Bopp (2003) para os ADRs de empresas latino-americanas negociados na NYSE (8,8\% no ano), incluindo ADRs de empresas brasileiras, há cerca de uma década.

\section{TABELA 7. Modelos estimados para relacionar o retorno à PIN - 2011}

\begin{tabular}{|c|c|c|c|c|c|c|}
\hline \multirow{2}{*}{ Variável } & \multicolumn{3}{|c|}{ Original } & \multicolumn{3}{|c|}{ Adaptado } \\
\hline & Coeficiente & Estatística t & Significância+ & Coeficiente & Estatística t & Significância ${ }^{+}$ \\
\hline Constante & 7,353 & 1,252 & 0,211 & $-13,568$ & $-1,613$ & 0,107 \\
\hline$\beta$ & $-6,928$ & $-3,776$ & $0,000^{\star}$ & $-7,696$ & $-3,688$ & $0,000^{*}$ \\
\hline PIN & 0,168 & 2,068 & $0,039^{\star \star}$ & 0,195 & 2,361 & $0,019^{\star \star}$ \\
\hline TAM & 0,142 & 0,359 & 0,720 & 1,352 & 2,577 & $0,010^{*}$ \\
\hline BM & $-3,305$ & $-4,345$ & $0,000^{\star}$ & $-3,324$ & $-4,394$ & $0,000^{*}$ \\
\hline Liq & & & & $-2,920$ & $-2,681$ & $0,008^{*}$ \\
\hline Volat & & & & 0,211 & 2,423 & $0,016^{\star *}$ \\
\hline \multicolumn{3}{|l|}{ Observações } & 792 & & & 792 \\
\hline \multicolumn{3}{|l|}{ Estatística F } & $11,148^{*}$ & & & $9,543^{*}$ \\
\hline \multicolumn{3}{|l|}{$\mathrm{R}^{2}$} & 0,054 & & & 0,068 \\
\hline \multicolumn{3}{|l|}{$\mathrm{R}^{2}$ Ajustado } & 0,049 & & & 0,061 \\
\hline
\end{tabular}

Notas: + Estimados com correção de White para heteroscedasticidade, * significante a $1 \%$ e ${ }^{\star \star}$ significante a $5 \%$.

No segundo modelo, denominado "adaptado", foram incluídas as variáveis liquidez e volatilidade. Com isso, pode-se verificar que o poder explicativo do modelo aumentou ( $R^{2}$ ajustado passou de 0,049 para 0,061), que ambas as variáveis incluídas foram significantes para a determinação do retorno durante o período analisado e que essa inclusão influenciou a relação do retorno com o tamanho das firmas, que passou a ser significante a $1 \%$. Destaque seja dado às variáveis tamanho, que teve seu coeficiente significativamente aumentado, e liquidez, que apresentou coeficiente negativo $(-2,920)$, indicando que seu aumento significou redução do retorno das ações. Como observa Carvalho (2002), no mercado brasileiro, certamente essas duas variáveis estão intrinsecamente relaciona- das, tendo em vista as características desse mercado, onde algumas das ações mais líquidas são as ações preferenciais das maiores companhias do mercado, que nem sempre apresentam os maiores retornos.

No que diz respeito à precificação da PIN no modelo adaptado, nota-se que a inclusão das variáveis liquidez e volatilidade, diretamente relacionadas à assimetria de informação nesse mercado, contribuiu com o aumento do coeficiente da variável $P I N$, sugerindo que um aumento de $10,0 \%$ na $P I N$ pode ocasionar um aumento no retorno das ações de 1,95\% no trimestre, ou 8,0\% no ano. Dessa forma, nota-se que o mercado de capitais brasileiro possui um prêmio pelo risco de informação. Com isso, as evidências desse estudo ratificam as ob- 
servações de Duarte e Young (2009), de que os mercados de países emergentes tendem a apresentar maior assimetria de informação, e de Easley, Hvidkjaer e O’Hara (2002), de que o retorno aumenta quando a assimetria de informação aumenta. Apesar disso, alerta-se que essas evidências podem estar sendo influenciadas por fenômenos não capturados pelos modelos utilizados, que nada mais são do que simplificações de uma realidade complexa. Por isso, é importante relembrar que os resultados aqui apresentados se limitam à amostra analisada.

\section{CONSIDERAÇÕES FINAIS E LIMITAÇÕES DO ESTUDO}

Apesar de a assimetria de informação ser um tema recorrente entre os estudos que abordam o mercado acionário brasileiro e de a PIN ser uma metodologia de mensuração de assimetria largamente utilizada em pesquisas no exterior, raros são os estudos que aplicam essa metodologia ao mercado de capitais brasileiro. Nesse sentido, a principal contribuição deste estudo é a aplicação desse modelo econômico ao mercado nacional, apresentando consistentes evidências empíricas sobre a existência de negociações com informação privilegiada no mercado nacional, tendo por base um conjunto de informações em alta frequência sobre as próprias negociações das ações desse mercado.

Em se tratando dessas evidências, pode-se observar que, apesar da existência de legislação específica criminalizando a utilização de informação privilegiada na negociação de ações no mercado acionário brasileiro, os resultados deste estudo reforçam os indícios de que operadores informados tenham realizado transações nesse mercado. Em média, pode-se verificar que há uma probabilidade de 53,0\% de terem ocorrido eventos informacionais durante o período analisado. Além disso, aproximadamente $49,2 \%$ desses eventos podem ter apresentado sinal positivo, indicando o surgimento de boas notícias. Tendo em vista o momento atual vivido pela economia brasileira e por seu mercado de capitais, que têm apresentado um significativo crescimento na última década, é preocupante observar que as más notícias tenham sobressaído às boas. Contudo, não se pode refutar a possibilidade de esse resultado ter sido influenciado pelo período pós-crise econômica de 2008/2009.

0 mesmo pode ser dito para a PIN observada, indicando que $22,9 \%$ das transações ocorridas nesse período podem ter sido baseadas em informação. Isso sugere que, durante o período analisado, a HME, em sua forma forte, não foi válida para o mercado brasileiro. Ainda, percebe-se que as ações que apresentaram menor assimetria de informação foram aquelas lista- das nos segmentos com maiores exigências de governança, especialmente aquelas do Novo Mercado. Entretanto, as ações do Nível 1 apresentaram maior PIN média que as ações do mercado tradicional, que, a priori, não possuem práticas diferenciadas de governança. Com isso, infere-se que o molde atual desse nível diferenciado de governança corporativa pode não estar adequado à redução da assimetria de informação existente nas transações com suas ações.

No que diz respeito à relação dessa assimetria com o retorno das ações, verificou-se que as variáveis $P I N$ e retorno apresentaram relação positiva e significante estatisticamente, como esperado. Assim, pode-se verificar que um aumento de $10,0 \%$ na PIN proporcionou um aumento médio anual de $8,0 \%$ no retorno das ações, valor superior àquele apurado por Easley, Hvidkjaer e O'Hara (2002) para o mercado norte-americano, o que sugere que o mercado de capitais brasileiro paga um prêmio pelo risco de informação superior ao mercado dos Estados Unidos. Por fim, vale observar que os resultados aqui apresentados se restringem às ações analisadas. Por isso, não podem ser generalizados, pois representam apenas um recorte da realidade em questão. Contudo, atenta-se que essas limitações não invalidam o estudo.

\section{REFERÊNCIAS}

ABAD, D; RUBIA, A. Modelos de estimación de la probabilidad de negociación informada: una comparación metodológica en el mercado español. Revista de Economía Financeira, n. 7, p. 26-53, 2005.

ALBANEZ, T; VALLE, M. R. Impactos da assimetria de informação na estrutura de capital de empresas abertas. Revista Contabilidade \& Finanças, v. 20, n. 51, p. 6-27, 2009.

BARBEDO, C. H; SILVA, E. C; LEAL, R. P. C. Probabilidade de informação privilegiada no mercado de ações, liquidez intradiária e níveis de governança corporativa. Revista Brasileira de Economia, v. 63, n. 1, p. 49-60, 2009.

BOPP, E. Negociação com informação diferenciada em ADRs da América Latina. 2003. 53 f. Dissertação de Mestrado em Economia, Escola de Pós-Graduação em Economia da Fundação Getulio Vargas, Rio de Janeiro, 2003.

BRASIL. Lei no 6.385, de 7 de setembro de 1976. Dispõe sobre o mercado de valores mobiliários e cria a Comissão de Valores Mobiliários. Disponível em: 〈http://www.planalto.gov.br/ccivil_03/Leis/L6385.htm〉. Acesso em 07.11.2012.

BRASIL. Lei $n^{0}$ 10.303, de 31 de outubro de 2001. Altera e acrescenta dispositivos na Lei no $6.404 / 76$, que dispõe sobre as Sociedades por Ações, e na Lei no $6.385 / 76$, que dispõe sobre o mercado de valores mobiliários. Disponível em: 〈http://www.planalto.gov.br/ccivil_03/ Leis/LEIS_2001/L10303.htm〉. Acesso em 07.11.2012.

CAMARGOS, M. A; BARBOSA, F. V. Teoria e evidência da eficiência informacional do mercado de capitais brasileiro. Caderno de Pesquisas em Administração, v. 10, n. 1, p. 41-55, 2003. 
CARVALHO, A. G. Governança corporativa no Brasil em perspectiva. Revista de Administração da USP, v. 37, n. 3, p. 19-32, 2002.

CRUCES, J. J; KAWAMURA, E. Insider trading and corporate governance in Latin America. Inter-American Development Bank, Research Network Working Paper R-513, 2005.

DUARTE, J; YOUNG, L. Why is PIN priced? Journal of Financial Economics, V. 91, n. 2, p. 119-138, 2009.

EASLEY, D; KIEFER, N. M; O’HARA, M; PAPERMAN, J. B. Liquidity, information and infrequently traded stocks. The Journal of Finance, v. 51, n. 4, p. 1405-1436, 1996.

EASLEY, D; KIEFER, N. M; O'HARA, M. One day in the life of a very common stock. Review of Financial Studies, v. 10, n. 3, p. 805-835, 1997.

EASLEY, D; HVIDKJAER, S; O'HARA, M. Is information risk determinant of asset returns? The Journal of Finance, v. 57, n. 5, p. 2185-2221, 2002.

EASLEY, D; HVIDKJAER, S; O'HARA, M. Factoring information into returns. Journal of Financial and Quantitative Analysis, v. 45, n. 2, p. 293-309, 2010.

EASLEY, D; O'HARA, M. Price, trade size, and information in securities markets. The Journal of Financial Economics, v. 19, n. 1, p. 69-90, 1987.

EASLEY, D; O'HARA, M. Time and the process of security price adjustment. The Journal of Finance, v. 47, n. 2, p. 577-605, 1992.

FAMA, E. F. Efficient capital markets: a review of theory and empirical work. The Journal of Finance, v. 25, n. 2, p. 383-417, 1970.
FAMA, E. F. Efficient capital markets: II. The Journal of Finance, v. 46, n. 5, p. 1575-1617, 1991.

FAMA, E. F; MACBETH, J. D. Risk, return, and equilibrium: empirical tests. Journal of Political Economy, v. 81, n. 3, p. 607-636, 1973.

HALOV, N; HEIDER, F. Capital structure, risk and asymmetric information. Quarterly Journal of Finance, v. 1, n. 4, p. 767-809, 2011.

HEIDLE, H. G; HUANG, R. D. Information-based trading in dealer and auction markets: an analysis of exchange listings. Journal of Financial and Quantitative Analysis, v. 37, n. 3, p. 391-424, 2002.

JENSEN, M. C; MECKLING, W. H. Theory of the firm: managerial behavior, agency costs and ownership structure. Journal of Financial Economics, v. 3, n. 4 , p. 305-360, 1976.

LEE, C. M. C; READY, M. J. Inferring trade direction from intraday data. The Journal of Finance, v. 46, n. 2, p. 733-746, 1991.

NYHOLM, K. Estimating the probability of informed trading. The Journal of Financial Research, v. 25, n. 4, p. 485-505, 2002.

NYHOLM, K. Inferring the private information content of trades: a regime-switching approach. Journal of Applied Econometrics, v. 18, n. 4, p. $457-470,2003$.

VIEIRA, S. P; MENDES, A. G. S. T. Governança corporativa: uma análise de sua evolução e impactos no mercado de capitais brasileiro. Organizações em Contexto, v. 2, n. 3, p. 48-67, 2006. 